\title{
Infrared external reflection spectra of Janus gold nanoparticle monolayers prepared at the air-water interface
}

\author{
H. Sakai ${ }^{1, *}, A$. Matsuzawa ${ }^{1}$ and $T$. Kawai $^{2}$ \\ ${ }^{1}$ National Institute of Technology, Oyama College, 771 Nakakuki, Ooaza, Oyama, Tochigi 323-0806, Japan \\ ${ }^{2}$ Tokyo University of Science, Kagurazaka 1-3, Shinjuku-ku, Tokyo 162-8601, Japan
}

\begin{abstract}
Nanoparticle monolayers at the air-water interface have received broad attention because of their potential applications owing to the tunability of their surface properties. We prepared gold nanoparticle monolayers by adsorption of hydrophilized nanoparticles from solution onto Langmuir monolayers of amphiphilic organic molecules (polyion complexes of deuterated stearic acid and polyallylamine) at the airwater interface, followed by adsorption of dodecyltrimethylammonium bromide onto the underside of these particles. The infrared external reflection spectra of the monolayers recorded both during and after preparation showed that the adsorption of gold nanoparticles on the Langmuir monolayers and that of dodecyltrimethylammonium bromide on the nanoparticles occurred in several tens of minutes. The infrared spectra also showed that both stearic acid and dodecyltrimethylammonium bromide molecules were highly ordered on the gold nanoparticles at the air-water interface. These results suggested that this preparation procedure is a useful fabrication method for Janus particles.
\end{abstract}

\section{Introduction}

The construction of 2D arrays of nanoparticles (NPs) is of great importance owing to their potential applications in electronic, magnetic, and optical devices [1-3]. Gold nanoparticle (AuNP) monolayers at the air-water interface have recently attracted widespread attention because of their tunable lateral surface density and ability to form 3D assemblies via the Langmuir-Blodgett method [4-17]. Moreover, NP monolayers can easily form anisotropic structures at the air-water interface because they are vertically confined. However, AuNP monolayers prepared by adsorption of hydrophilized AuNPs from solution onto Langmuir monolayers of amphiphilic organic molecules at the air-water interface have not been examined in as much detail as those prepared by spreading highly hydrophobized NPs over the air-water interface. The preparation method involving hydrophilized AuNPs allows for the fabrication of Janus particles through the addition of different ligands to the underside of particles partially covered with molecules in the Langmuir monolayer. Moreover, this method has the advantage of using little organic solvent during the fabrication of Janus particles. To the best of our knowledge, few studies have used this method to fabricate Janus particles [18]. It is therefore important to determine the adsorption kinetics and the structural arrangement of the ligands on the nanoparticles prepared using this method.
In this study, we prepared AuNP monolayers covered with dodecyltrimethylammonium bromide (DTAB) and Langmuir monolayers of polyion complexes of deuterated stearic acid (d-STA) and polyallylamine (PAA) at the air-water interface (Figure 1). The infrared external reflection (IER) spectra of the monolayers were recorded in situ. The structural arrangement and configurations of the molecules was estimated from the intensity and wavenumber of the band peaks in the IER spectra. We also observed the adsorption of the molecules on the nanoparticles during the preparation process.

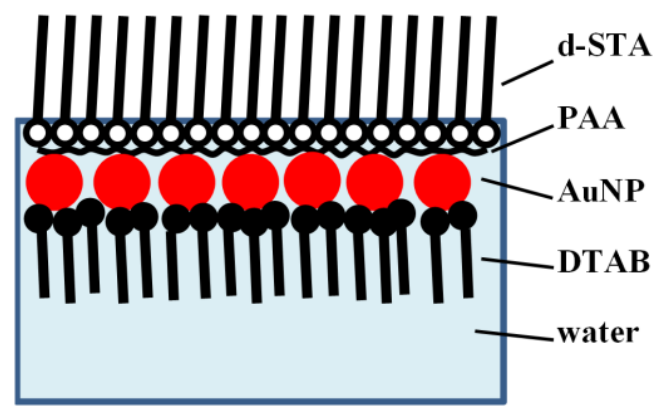

Fig. 1. Schematic illustration of AuNP monolayers covered with DTAB and Langmuir monolayers of d-STA and PAA.

\footnotetext{
*Corresponding author: sakai@oyama-ct.ac.jp
} 


\section{Materials and Methods}

\subsection{Materials}

Hydrogen tetrachloroaurate(III) trihydrate (SigmaAldrich), trisodium citrate dehydrate (Cica), PAA (Nitto Boseki, MW: 8000), and d-STA (CDN ISOTOPES) were used without further purification. The deuterated compound was used so that we can differentiate between d-STA and DTAB in the IER spectra. DTAB (SigmaAldrich) was recrystallized from an acetone solution.

\subsection{Preparation Method}

AuNPs were prepared according to the standard sodium citrate reduction method [19]. Transmission electron microscopy (TEM) observations and dynamic light scattering measurements revealed that the mean diameter of the AuNPs was around $20 \mathrm{~nm}$. The concentration of the AuNP solution was calculated to be $3.0 \times 10^{15}$ particles/L.

AuNP monolayers at the air-water interface were prepared by adsorption of AuNPs onto Langmuir monolayers of polyion complexes of d-STA and PAA. The polyion complex was prepared in methanol at a molar ratio of 1:2 (d-STA:PAA, monomer units). A chloroform solution of the polyion complex was then spread onto the AuNP solution surface in a Langmuir trough to give a Langmuir monolayer with the surface area of $0.20 \mathrm{~nm}^{2} / \mathrm{d}-\mathrm{STA}$ molecule. After waiting $60 \mathrm{~min}$ to allow the AuNPs to adsorb onto the Langmuir monolayer, the AuNP solution was replaced with water as follows: $1 \mathrm{~mL}$ of the AuNP solution underneath the Langmuir monolayer was removed from the Langmuir trough $(12 \mathrm{~mL})$ using a pipette, and then $1 \mathrm{~mL}$ of water was added into the trough. This process was repeated 100 times. Subsequently, a DTAB solution was injected into the Langmuir trough. The concentration of DTAB was about $8 \mathrm{mM}$, which is less than the critical micelle concentration of DTAB. After waiting $60 \mathrm{~min}$ to allow DTAB molecules to adsorb onto the AuNP monolayer, the DTAB solution was replaced with water in the manner described above. The final concentration of DTAB in the trough was calculated to be about 0.001 mM.

\subsection{IER}

The IER spectra during and after the preparation of the NP monolayer were recorded at the air-water interface using a Nicolet iS50 FT-IR spectrophotometer equipped with a mercury-cadmium-telluride (MCT) detector with a resolution of $4 \mathrm{~cm}^{-1}$. A trough with an $80 \times 22 \mathrm{~mm}^{2}$ effective surface area attached to a Specac 19650 monolayer/grazing angle accessory was used. The incident angle from the surface normal was $40^{\circ}$. An unpolarized beam was used, and 500 interferograms were collected. The vertical axis of the reflection spectrum was defined as $\log \left(\mathrm{R}_{0} / \mathrm{R}\right)$, where $\mathrm{R}$ and $\mathrm{R}_{0}$ are the sample and background reflectance, respectively. The background reflectance was recorded on the surface of either AuNP solution or water, according to the subphase of the each sample.

\subsection{TEM}

AuNP monolayer samples for TEM measurements were fabricated on a 250 mesh carbon-covered $\mathrm{Cu}$ grid (Okenshoji ELS-C10) using a horizontal method [8]. The samples were examined using a JEOL JEM-1011 transmission electron microscope operating at $100 \mathrm{kV}$.

\section{Results and Discussion}

Figure 2(a) shows the TEM image of a TEM sample that was fabricated $60 \mathrm{~min}$ after spreading the polyion complex solution on the AuNP solution. The AuNPs formed a monolayer underneath the Langmuir monolayer of the polyion complex. Hereafter, we designate this monolayer as a d-STA/PAA/AuNP film. It has been reported that AuNPs interact with ionic molecules in Langmuir monolayers through ionic bonding to form monolayers of AuNPs [4, 6-9, 15, 17]. The present result indicates that ionic bonding likely occurred between the $\mathrm{NH}_{3}{ }^{+}$and $\mathrm{COO}^{-}$groups of PAA and AuNPs, respectively.
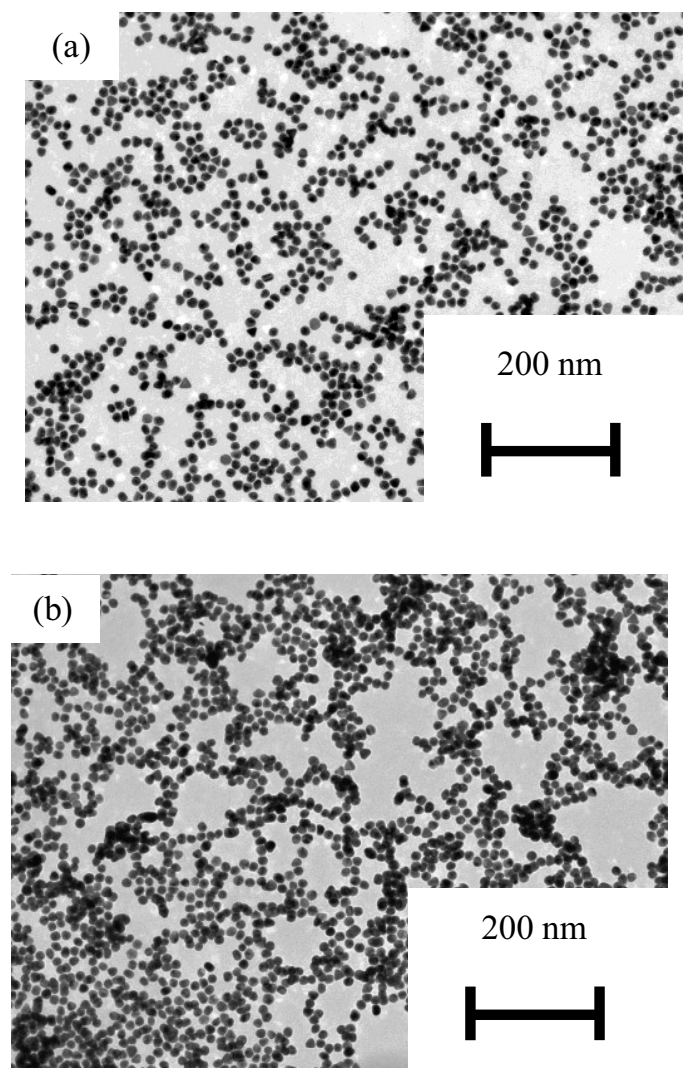

Fig. 2. TEM images: (a) STA/PAA/AuNP film before replacement of the AuNP solution with water and (b) dSTA/PAA/AuNP/DTAB film after replacement of the DTAB solution with water.

Figure 2(b) shows the TEM image of a TEM sample that was fabricated after replacing the DTAB solution with water. We designate this film as d- 
STA/PAA/AuNP/DTAB. The film remained on the water surface, even after replacing the solution (AuNP and DTAB) with water twice. To the best of our knowledge, there have been few reports about AuNP monolayers subjected to such harsh treatments. The outstanding stability of the present film can probably be attributed to the strong ionic bonding interactions and the robustness of the polyion complex in the Langmuir monolayer.

Figure 3 shows the IER spectra of the $d$ STA/PAA/AuNP film on the AuNP solution and water in the $\mathrm{CH}_{2}$ and $\mathrm{CD}_{2}$ stretching vibrational region. The times shown in this figure represent the elapsed time after spreading the polyion complex solution on the surface of the AuNP solution. The peaks corresponding to the antisymmetric and symmetric $\mathrm{CD}_{2}$ stretching band of $\mathrm{d}-$ STA are observed in the IER spectra. Figure 4 shows a plot of the intensity (peak height) of the antisymmetric $\mathrm{CD}_{2}$ stretching band against the elapsed time on the AuNP solution and on the water surface. On the AuNP solution, the absorbance increases for several tens of minutes, probably owing to surface-enhanced infrared absorption [20] because of adsorption of AuNPs underneath the Langmuir monolayer. This result indicates that the d-STA/PAA/AuNP film was formed in several tens of minutes and then remained intact. The appearance of this band at $2193 \mathrm{~cm}^{-1}$ indicated that the conformation of the d-STA molecule was all-trans [21]. The intensity and wavenumber of this band remained largely unchanged after replacing the AuNP solution with water.

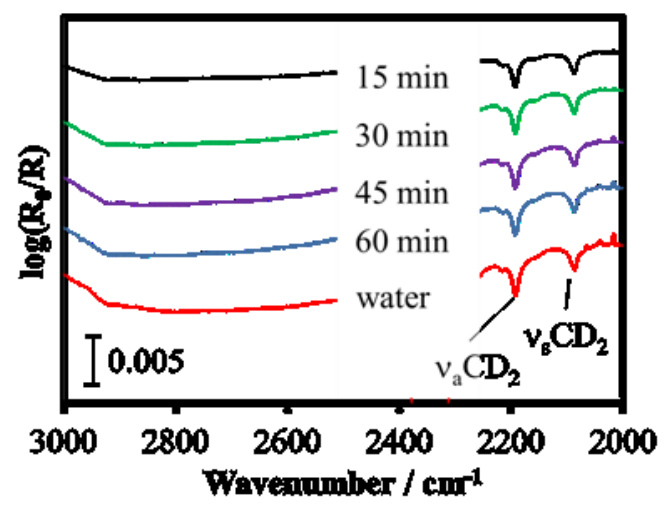

Fig. 3. IER spectra of the d-STA/PAA/AuNP film on the AuNP solution and water.

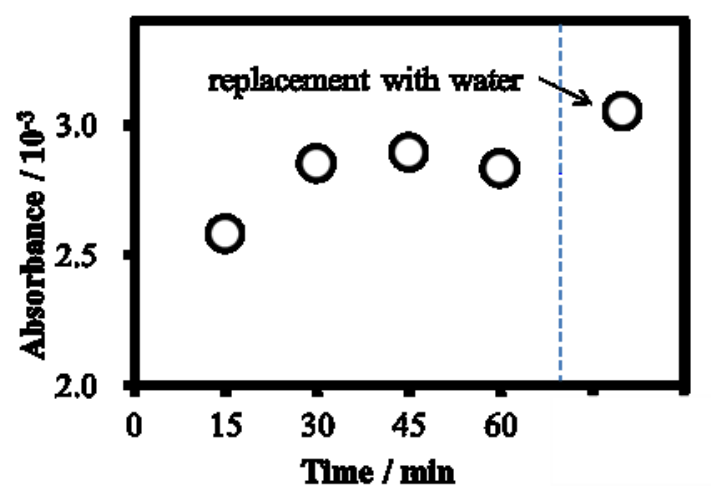

Fig. 4. $v_{\mathrm{a}} \mathrm{CD}_{2}$ absorbance vs. time on the AuNP solution and water.

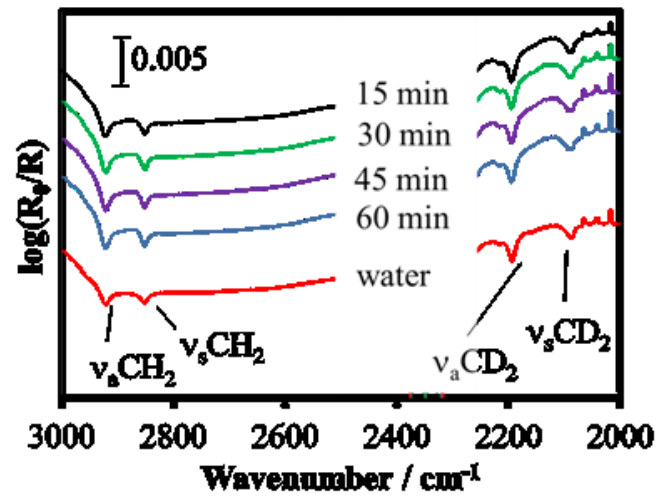

Fig. 5. IER spectra of the d-STA/PAA/AuNP/DTAB film on the DTAB solution and water.

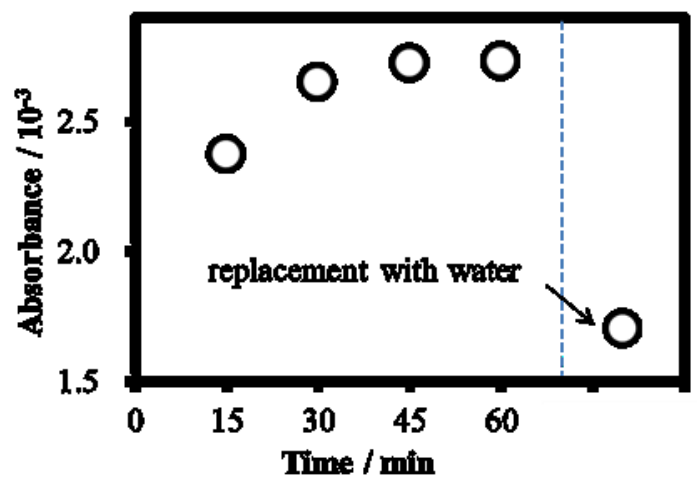

Fig. 6. $v_{\mathrm{a}} \mathrm{CH}_{2}$ absorbance vs. time on the DTAB solution and water.

Figure 5 shows the IER spectra of the dSTA/PAA/AuNP/DTAB film on the DTAB solution and water. The times shown in this figure represent the elapsed time after injecting the DTAB solution into the water underneath the d-STA/PAA/AuNP film. The peaks corresponding to the $\mathrm{CD}_{2}$ stretching bands of d-STA and the $\mathrm{CH}_{2}$ stretching bands of DTAB can be observed in the IER spectra. Figure 6 shows a plot of the intensity of the antisymmetric $\mathrm{CH}_{2}$ stretching band of DTAB against the elapsed time on the DTAB solution and on water. Upon adding DTAB molecules to the water, the peak corresponding to DTAB appeared quickly and continued to gradually increase for several tens of minutes. This result indicates that DTAB was quickly adsorbed underneath the AuNPs, with saturation reached after several tens of minutes. The DTAB peak persisted even after replacing the DTAB solution with water, although the intensity decreased. This decrease is probably associated with the elimination of extra DTAB molecules that were not adsorbed on the AuNPs. However, the retention of this peak indicates that the DTAB molecules adsorbed tightly onto the AuNPs through ionic bonding between the quaternary ammonium ion and carboxylate ion groups of DTAB and AuNPs, respectively. The wavenumber of the antisymmetric $\mathrm{CH}_{2}$ stretching band in the d-STA/PAA/AuNP/DTAB film at $2921 \mathrm{~cm}^{-1}$ was lower than that of DTAB molecules at the air-water interface $\left(2924 \mathrm{~cm}^{-1}\right)$; this indicates that DTAB molecules are more ordered on AuNPs than on the water 
surface. On the other hand, following addition of DTAB, the intensity of the d-STA band decreased slightly and the wavenumber increased $\left(2194 \mathrm{~cm}^{-1}\right)$, indicating that $\mathrm{d}-$ STA molecules on the AuNPs became somewhat disordered on adding DTAB. However, as the increase in the wavenumber is small and there is little difference between the TEM images before and after adding DTAB, we believe that Janus nanoparticles could be prepared properly using the present method.

We were able to prepare Janus particles with two kinds of ligands spatially separated at the air-water interface by means of a mild (using little organic solvent) procedure. The robustness of the polyion complex in the Langmuir monolayer likely enables this procedure. We could also estimate the structural arrangement of the ligands during and after the preparation of the monolayer. These spectral results indicate that this preparation method can be used to successfully fabricate Janus particles.

\section{Conclusions}

In this study, we prepared AuNP monolayers covered with DTAB and Langmuir monolayers of polyion complexes of d-STA and PAA at the air-water interface. The IER spectra of the monolayers reordered during and after preparation of the monolayer confirmed that the adsorption of both d-STA and DTAB molecules on the AuNPs is completed in several tens of minutes. Moreover, the two ligands are arranged in a highly ordered fashion on the particles. While further work using other ligands needs to be discussed, this preparation method is a useful mild procedure for fabricating Janus particles.

\section{References}

1. M. C. M. Daniel, D. Astruc, Chem. Rev. 104, 293 (2004)

2. A. R. Tao, J. Huang, P. Yang, Acc. Chem. Res. 41, $1662(2008)$

3. D. V. Talapin, J.-S. Lee, M. V. Kovalenko, E. V. Shevchenko, Chem. Rev. 110, 389 (2010)

4. T. Kawai, D. J. Neivandt, P. B. Davies, J. Am. Chem. Soc. 122, 12031 (2000)

5. T. Hassenkam, K. Nørgaard, L. Iversen, C.J. Kiely, M. Brust, T. Bjørnholm, Adv. Mater. 14, 1126 (2002)

6. K. M. Mayya, A. Gole, N. Jain, S. Phadtare, D. Langevin, M. Sastry, Langmuir. 19, 9147 (2003)

7. K. M. Mayya, N. Jain, A. Gole, D. Langevin, M. Sastry, J. Colloid Interface Sci. 270, 133 (2004)

8. S. Pang, O. Tetsuya, W. Tomoyuki, T. Kondo, T. Kawai, J. Colloid Interface Sci. 285, 634 (2005)

9. L. -H. Chen, A. Dudek, Y. -L. Lee, C. -H. Chang, Langmuir. 23, 3123 (2007)

10. A. Mogilevsky, R. Jelinek, Langmuir. 27, 1260 (2011)

11. S. Tatur, A. Badia, Langmuir. 28, 628 (2012)

12. K. D. Comeau, M. V. Meli, Langmuir. 28, 377 (2012)
13. V. Sashuk, R. Hołyst, T. Wojciechowski, M. Fiałkowski, J. Colloid Interface Sci. 375, 180 (2012)

14. D. I. Babenko, A. A. Ezhov, D. S. Turygin, V. K. Ivanov, V. V. Arslanov, M. A. Kalinina, Langmuir. 28, 125 (2012)

15. A. A. Torrano, Â. S. Pereira, O. N. Oliveira, A. Barros-Timmons, Colloids Surfaces B Biointerfaces. 108, 120 (2013)

16. B. P. Gagnon, M. V. Meli, Langmuir. 30, 179 (2014)

17. S. Srivastava, D. Nykypanchuk, M. Fukuto, O. Gang, Nano Lett. 8, 9857 (2014)

18. A. Walther, A. H. E. Müller, Chem. Rev. 113, 5194 (2013)

19. G. Frens, Nat. Phys. Sci. 241, 20 (1973)

20. M. Osawa, K. Ataka, K. Yoshii, Y. Nishikawa, Appl. Spectrosc. 47, 1497 (1993)

21. E. Le Calvez, D. Blaudez, T. Buffeteau, B. Desbat, E, Langmuir. 17, 670 (2001) 